\title{
Analytical modelling prediction by using wake oscillator model for vortex-induced vibrations
}

\author{
W.N.W. Hussin ${ }^{1}$, F.N. Harun ${ }^{2}$, M.H. Mohd ${ }^{1}$ and M.A.A. Rahman ${ }^{1 *}$ \\ ${ }^{1}$ School of Ocean Engineering, Universiti Malaysia Terengganu, \\ Terengganu, Malaysia \\ *Email: mohdasamudin@umt.edu.my \\ Phone: +6096683698; Fax: +6096683991 \\ ${ }^{2}$ School of Informatics and Applied Mathematics, \\ Terengganu, Malaysia
}

\begin{abstract}
Explorations by oil and gas companies have moved from shallow to deep water to satisfy consumers' changing needs. A circular- shaped cylinder structure placed in deep water is also known spar platform, is normally floated and subjected to severe conditions which cause unpleasant motions and fatigue damage to the structure. Wake oscillator model is considered a semi-empirical model that is most suitable for the evaluation of Vortexinduced Vibrations (VIV) structure features during the design stage. This work focuses on validation of wake oscillator model for VIV with previously semi-empirical method. Wake oscillator and structure oscillator models are coupled based on the Van der Pol Equation. The coupled models were evaluated and analytically validated. The results showed a qualitative and quantitative agreement with previous analytical, at the same maximum peak amplitude response, $y_{0}=0.22$, with $1.2 \%$ percentage error . Meanwhile, experimental results from literature only showed a qualitative agreement but not in quantitative agreement. Analytical modelling may offer potential means for investigating VIV features and provide a fundamental analysis by using a cost saving but reliable method. Further works should be done to characterise the VIV and Vortex-induced Motions phenomenon for spar platform.
\end{abstract}

Keywords: vortex-induced vibrations; wake oscillator model, circular cylinder; van der pol equation; vortex-induced motions.

\section{INTRODUCTION}

Since the past few decades' oil and gas industry has become one of the most prominent and leading industries. The maturation of this industry is represented by the increasing number of offshore structures around the world due to the price and increased demand for fuel every day. Williamson \& Govardhan [1] stated that Vortex-induced vibrations (VIV) is a critical problem in the industry for immersed structure design such as a spar platform. Researchers have investigated and developed new technologies and techniques for better prediction of the VIV structure that are undergoing hydrodynamic forces (wave and current) so as to minimize damages to the structure. VIV has become one of the major problems faced by companies in dealing with the increasing cost of building a platform. That is because a large safety factor is applied during the design stage due to lack of knowledge and understanding on VIV prediction. Extensive study about VIV was done 
for a few decades, such as [2] [3-13], and thus attempts to reduce the VIV of structures have been done. It is important to know that many object shapes may lead to VIV. However, this work is limited to observation based on circular-shaped cylinder (spar platform). It is well known that VIV can limit the structural life expectancy of materials and may even lead to catastrophic failures [14]. One of the examples of catastrophic failures happened in 1940, where the Tacoma-Narrow Bridge collapsed due to severe wind-induced vibrations [15]. Some recent investigation on VIV phenomenon based on circular cylindrical are seen in [16]. VIV is one of the important considerations for offshore platforms design. Construction and maintenance costs have increased due to large safety factors being applied in the structure design as a result of insufficient understanding of fluid-structure interactions. There are other priorities that need to be catered for during the preliminary design stage, such as providing a fundamental analysis by using a reduced-cost but reliable method. It is envisaged that through this proposed work a critical area to be tackled and become useful contributions are worth mentioning in earlier discussion. Although a great deal of effort was made during recent decades to improve VIV of ocean structures based on analytical, numerical and experimental approaches that are used for predicting vibrations due to vortex shedding, the available analytical approach are still rather crude.

Generally, many topics based on VIV phenomenon were extensively reviewed and many successful approaches were based on numerical simulations and experimental techniques but not many are done analytically. The objectives of this paper are to further investigate the response of the cylinder to forces due to fluid flow by using analytical modelling and provide knowledge to the understanding of VIV phenomenon, and also give significant benefits to the oil and gas industry. As for the analytical way, is appropriate to use the wake oscillator model, which is also known as a semi-empirical model. Work with experienced structural VIV can be classified into three main types, one of them is wake-body (wake-oscillator) coupled models [17]. This type is coupled through common terms in equations for the structure and the wake oscillations. Earlier studies based on wake oscillator model were done by [18-20] and [21]. This model can illustrate most of the VIV features caused by the vortex. One of the features is the lockin domain. This model will be discussed in detail in other sections. The result from this model will be validated with the previous model, then compared with experimental results from the literature.

\section{METHODS AND MATERIALS}

\section{Formulation of the Model}

The bluff body surface experiences a fluctuating pressure variation with time as the body is under the action of a fluid flow with velocity $U$. When a successive separation of vortices on each of its side takes place, oscillating pressure fields develop on the body. If the body is free to move in transverse direction to the fluid flow, the resultant periodic net forces will interact with the structure movement originating the vortex shedding induced vibration [22]. The parameter values used in the present coupling model are as shown in Table 1.

\section{Structural Oscillator}

Figure 1(a) illustrates a spar platform submerged in water and transformed into an analytical model, also known a semi-empirical model. The analytical models are based on a 1DOF of a rigid circular cylinder of diameter (D) that was elastically mounted. 
Figure 1(b) shows that the cylinder is restricted in such a way that it can crosswise oscillate to a uniform flow of free stream velocity (U). The in plane form displacement (Y) of cylinder crosswise to fluid flow can be as stated by a linear oscillator as defined in [23].

Table 1. Parameter values.

\begin{tabular}{lc}
\hline \multicolumn{1}{c}{ Parameter } & Value \\
Diameter, $D$ & 0.0381 \\
Strouhal Number, $S_{t}$ & 0.2 \\
Van Der Pol Parameter, $\varepsilon$ & 0.3 \\
Scaling of the coupling force, $A$ & 12 \\
Damping Coefficient, $\gamma$ & 0.8 \\
\hline
\end{tabular}

(a)

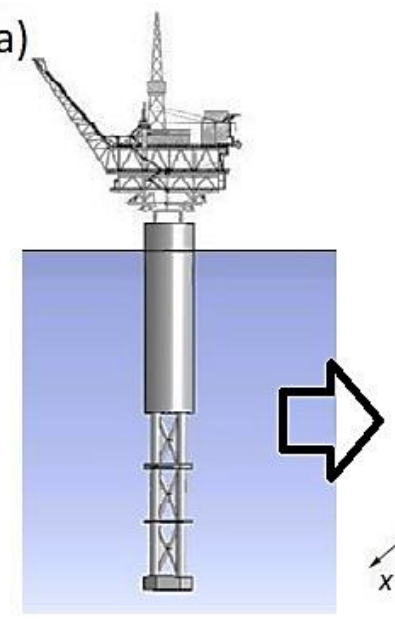

(b)

Figure 1. (a) Illustration of Spar Platform submerged in water[24]; (b) Illustration model of coupled structure and wake oscillator for 2D vortex-induced vibration [21] [23].

$$
m \ddot{Y}+r \dot{Y}+h Y=S
$$

where overdots (.) means derivatives with respect to dimensional time(T). This model the inviscid inertia effects of fluid [25] which is the mass $(m)$ consisting of two parts that is $m_{s}$, the mass of the structure, and the second one is $m_{f}$, the fluid-added mass by [23], and reads,

$$
m=m_{s}+m_{f}, \quad m_{f}=\frac{1}{4} C_{m} \rho D^{2} \pi, \quad \mu=\left(m_{s}+m_{f}\right) / \rho D^{2}
$$

where $\rho$ is the fluid density, $\mu$ is dimensionless mass ratio and $C_{m}$ is the added mass coefficient with assumption that a constant derived from a potential flow theory [25]. It reads $C_{m}=1$ at circular cross section case[23]. In Eq. (1), the linear damping $r$ models both viscous dissipations in support, $r_{s}$ and fluid-added damping, $r_{f}$ [23], namely,

$$
r=r_{s}+r_{f}, \quad r_{f}=\frac{2 \pi s_{t} U}{D} \gamma \rho D^{2} \pi
$$

where $\gamma$ is a stall parameter and is a function of oscillation frequency related to the mean sectional drag coefficient of the structure [25]. In this case, Blevins [25], Pantazopoulos 
[26] and Facchinetti et al. [23][30] assumed that the amplified drag coefficient, that are constant with $C_{D}=2.0 . S_{t}$ is Strouhal number as at common practice in the sub-critical range $300<R e<1.5 \times 10^{5}$ that assumes $S_{t}=0.2$ [25], [26]. The fluid added damping coefficient $\gamma=0.8$ from Eq. (3). The stiffness coefficient in Eq. (1), h only relates to outer effects. Searching for simplicity, it is assumed that stall parameter, $\gamma$ remains constant. To develop a two dimensional model, oscillation of all mass parameters, damping and stiffness is defined as per unit length. The fluid hydrodynamic effects on structure consist of two parts, i.e. the basic fluid effects, $m_{f}$ and another one is $r_{f}$, that has direct influence on the structure by Equation (2) and Equation (3), while the effects of vortices that are modelled as a forcing term is defined by Facchinetti et al. [23] and it reads as,

$$
S=\frac{1}{2} \rho U^{2} D C_{L}
$$

where $C_{L}$ is fluctuating lift coefficient. Defining structural angular frequency $\Omega_{s}=$ $\sqrt{h / m}$ structure reduced damping $\xi=r_{s} / 2 m \Omega_{s}$ and vortex-shedding frequency $\Omega_{\mathrm{f}}=$ $2 \pi \mathrm{S}_{\mathrm{t}} \mathrm{U} / \mathrm{D}$ explained by Facchinetti et al. [23], so that Eq. (1) becomes

$$
\ddot{Y}+\left(2 \xi \Omega_{s}+\frac{\gamma}{\mu} \Omega_{f}\right) \dot{Y}+\Omega_{f}^{2} Y=\frac{s}{M}
$$

\section{Wake Oscillator}

Equation of wake in Equation (1) was used to couple with equation of wake structure in Equation (6). Nature fluctuations of the vortex street is modelled by a nonlinear oscillator complying to the Van der Pol Equation [33].

$$
\ddot{q}+\varepsilon \Omega_{f}\left(q^{2}-1\right) \dot{q}+\Omega_{f}^{2} q=F
$$

The dimensionless wake variable ( $q$ ) that is related to the fluctuating lift coefficient is considered as the main variable, and can be defined as $=2 C_{L} / C_{L O}$. where $C_{L}$ is instantaneous lift coefficient and $C_{L}$ is reference lift coefficient of a stationary cylinder under vortex shedding. The right hand side forcing term of Equation (6), F, models the effect of cylinder motion on the wake dynamics.

\section{Coupling of Wake and Structural Oscillators}

In order to get a more suitable method, the dimensionless time $t=\Omega_{s} T$ and space coordinate $y=Y / D$ was introduced by Facchinetti et al. [23]. Equation (5) and (6) lead to the coupled fluid-structure dynamical system

$$
\ddot{y}+\left(2 \xi \delta+\frac{\gamma}{\mu}\right) \dot{y}+\delta^{2} y=s, \quad \ddot{q}+\varepsilon\left(q^{2}-1\right) \dot{q}+q=f
$$

where $\delta=\Omega_{\mathrm{s}} / \Omega_{\mathrm{f}}$ is the reduced angular frequency of the structure and also related to $U_{r}=2 \pi U /\left(\Omega_{\mathrm{s}} D\right)$, reduced flow velocity by [23].

$$
\delta=\frac{\Omega_{\mathrm{s}}}{\frac{2 \pi \mathrm{S}_{\mathrm{t}} \mathrm{U}}{\mathrm{D}}}=\frac{\Omega_{\mathrm{s}} \mathrm{D}}{2 \pi \mathrm{S}_{\mathrm{t}} \mathrm{U}}=\frac{1}{\mathrm{~S}_{\mathrm{t}} \mathrm{U}_{\mathrm{r}}}
$$

and also $s=S /\left(D \Omega_{f}^{2} m\right)$, considering the definition of $\mathrm{q}$ and $\mathrm{S}$ becomes 


$$
s=M q, \quad M=\frac{\frac{C_{L 0}}{2}}{8 \pi^{2} S_{t} \mu}
$$

In the large range of $\mathrm{Re}$, the reference lift coefficient is defined as $C_{L 0}=0.3$ [25] [26]. The mass number, $M$ is derived from Equation (9) and becomes

$$
M=\frac{0.05}{\mu}
$$

Various researchers have used different coupling models such as displacement coupling $f=A y$, velocity coupling $f=A \dot{y}$ and acceleration coupling $f=A \ddot{y}$ to define this forcing term. This paper focuses on the use of an acceleration coupling that is $f=$ $A \ddot{y}$ because acceleration coupling was successfully analysed to model all features of VIV rather than displacement and velocity coupling [23]. Therefore, Equation (7) becomes,

$$
\begin{gathered}
\ddot{y}+\left(2 \xi \delta+\frac{\gamma}{\mu}\right) \dot{y}+\delta^{2} y=M q \\
\ddot{q}+\varepsilon\left(q^{2}-1\right) \dot{q}+q=A \ddot{y}
\end{gathered}
$$

A and $\varepsilon$ are the parameters derived from experimental data on the wake dynamics, typically $\mathrm{A}=12$ and $\varepsilon=0.3[23,25]$ [27]. Overdots (.) of Eq. (11) are derivatives with respect to dimensionless time, $t$. For the next section, we will investigate wake oscillator dynamics Equation (7) by considering a harmonic motion of dimensionless amplitude $y_{0}$ and $\omega$ angular frequency. Specifically $y=y_{0} \cos (\omega t)$ and the wake oscillator in Equation (7) is forced by $f$ depends on the coupling model. In case of acceleration coupling, it reads,

$$
f=-A \omega^{2} y_{0} \cos (\omega t)
$$

Then defining a reduced velocity on the forcing frequency as Facchinetti et al. (2004), it reads as

$$
U_{r}=\frac{2 \pi}{\omega \Omega_{f}} \frac{U}{D}=\frac{1}{\omega S_{t}}
$$

Here now, the acceleration models of coupling in the parameter space $\left(U_{r}, y_{0}\right)$ have been analysed on the response of the wake oscillator. A response is sought in the form of

$$
q=q_{0} \cos (\omega t+\psi)
$$

where $q_{0}$ is time independent amplitude and $\psi$ phase that can be substituted into Equation (7), whereby the main harmonic contribution of nonlinearities is also considered.

$$
q_{0}^{6}-8 q_{0}^{4}+16\left[1+\left(\frac{\omega^{2}-1}{\varepsilon \omega}\right)^{2}\right] q_{0}^{2}=16\left(\frac{\|f\|}{\varepsilon \omega}\right)^{2}
$$

In Equation (16), the amplitude of the forcing, namely $\|f\|$ and defining a reference lockin state by $\omega=1$ at $U_{r}=1 / S_{t}$ The vortex lift magnification factor with respect to a stationary structure, experiencing vortex shedding. Equation (16) is derived as the unique real root of the bi-cubic polynomial of $q_{0}$ and get $K=q_{0} / 2$, which reads 


$$
K=\left(\frac{X}{36}\right)^{1 / 3}+\left(\frac{4}{3 X}\right)^{1 / 3}
$$

with

$$
X=\left(9 \frac{A}{\varepsilon} y_{0}\right)+\sqrt{\left(9 \frac{A}{\varepsilon} y_{0}\right)^{2}-48}
$$

Analytically, the phase of the transfer function of the wake oscillator is derived as for amplitude in Equation (16) for the displacement, velocity and acceleration coupling models [23]. It reads as

$$
\psi=\theta, \quad \psi=\theta+\frac{\pi}{2}
$$

with

$$
\tan \theta=\frac{\varepsilon \omega}{\omega^{2}-1}\left(\frac{q_{0}^{2}}{4}-1\right)
$$

\section{Coupling System}

Here, the coupled fluid structure system will be considered by Equation (7) as substitution in order to find a solution in the form that was already described by [23]. It reads

$$
y=y_{0} \cos (\omega t), \quad q=q_{0} \cos (\omega t-\varphi)
$$

Substitution in the structure oscillator Equation (7) will obtain the amplitude and phase of the liner transfer function between structure displacement and fluid variable that will become

$$
\frac{y_{0}}{q_{0}}=\frac{M}{\sqrt{\left(\delta^{2}-\omega^{2}\right)^{2}+\left(2 \xi \delta+\frac{\gamma}{\mu}\right)^{2} \omega^{2}}}, \quad \tan \varphi=\frac{-\left(2 \xi \delta+\frac{\gamma}{\mu}\right) \omega}{\delta^{2}-\omega^{2}}
$$

Substituting with considering only main harmonic contribution of the nonlinearities in the wake oscillator, Equation (7) will obtain equations on $q_{0}$ and $\omega$ that are amplitude and angular frequency, respectively[23].

$$
\begin{gathered}
q_{0}=2 \sqrt{1+\frac{A M}{\varepsilon} \frac{C}{\left(\delta^{2}-\omega^{2}\right)^{2}+\left(2 \xi \delta+\frac{\gamma}{\mu}\right)^{2} \omega^{2}}} \\
\omega^{6}-\left[1+2 \delta^{2}-(2 \xi \delta+\gamma / \mu)^{2}\right] \omega^{4}-\left[-2 \delta^{2}+(2 \xi \delta+\gamma / \mu)^{2}-\delta^{4}\right] \omega^{2}-\delta^{4}+G=0
\end{gathered}
$$

where the coefficients $\mathrm{C}$ and $\mathrm{G}$ depend on coupling model for acceleration, and it reads

$$
C=\left(2 \xi \delta+\frac{\gamma}{\mu}\right) \omega^{2}, \quad G=A M\left(\omega^{2}-\delta^{2}\right) \omega^{2}
$$

The angular frequency, $\omega$ appears from Equation (24) that produces one or three positive real roots. Based on the solutions, angular frequency using coupling Equation (22) and Equation (23) will arise the amplitude $y_{0}$ of the structure motions. 


\section{RESULTS AND DISCUSSION}

In Figure 2, with a proposed value by Facchinetti et al. [23] by using least square interpolation, $A / \varepsilon=40$, with Van Der Pol parameter, $\varepsilon=0.3$ and the coupling of force scale, $A=12$ shows that $y_{0}>0.5$ higher imposed structure motions. Comparing with experimental data from previous literature shows that lift magnification factor, $K$ increases with response amplitude up to certain point, then becomes lessen by $y_{0}$ function by [26] [23, 28-30]. This happen because of the natural crosswise spacing of the near wake vortex street that makes $y_{0}$ huge $[23,31]$. The response model from Equation (17) and Equation (18) is most suitable with experimental data and is used to solve coupled wake oscillator model in this paper.

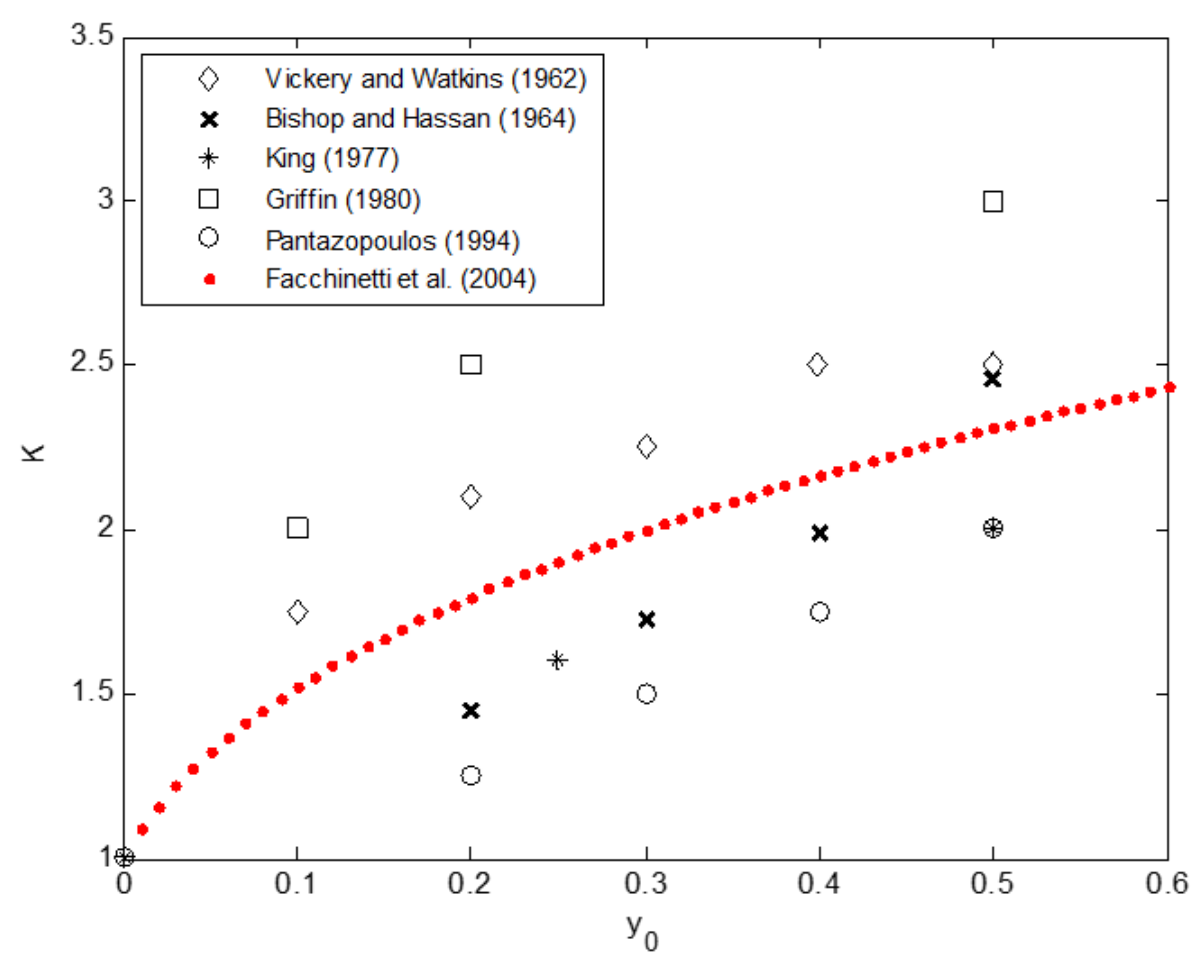

Figure 2. Lift magnification, $K$ as a function of the imposed structure motions amplitude, $y_{0}[23]$.

The lock-in phenomenon happens when there is vortex shedding that can cause large amplitude vibration, also called as vortex capturing. According to Bearman [32], the lock-in phenomenon occurs when the forcing frequency begins to unite with the shedding frequency. At this situation, the higher amplitude responses are monitored. This occurs when vortex shedding frequency turns out to be near to the natural frequency of crosswise vibrations of the structure [22]. As shown in Figure 3 and Figure 4, the reduced damping, $\xi=0.0031$ and scales of the effect of the wake structures, $M=0.0002$ are used to illustrate the case of uniform pivoted cylinder experiencing crosswise VIV in uniform flow by Balasubramanian et al.[33]. 


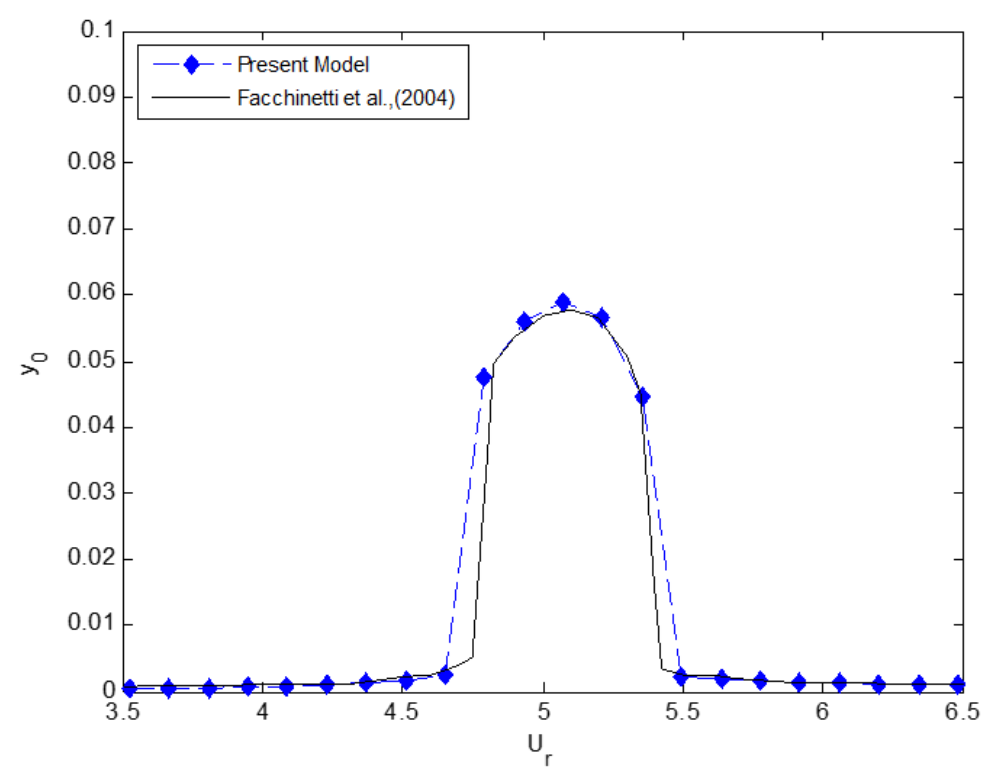

Figure 3. Amplitude of structure oscillator, $y_{0}$ using acceleration model as a function of reduced velocity, $U_{r}[23]$.

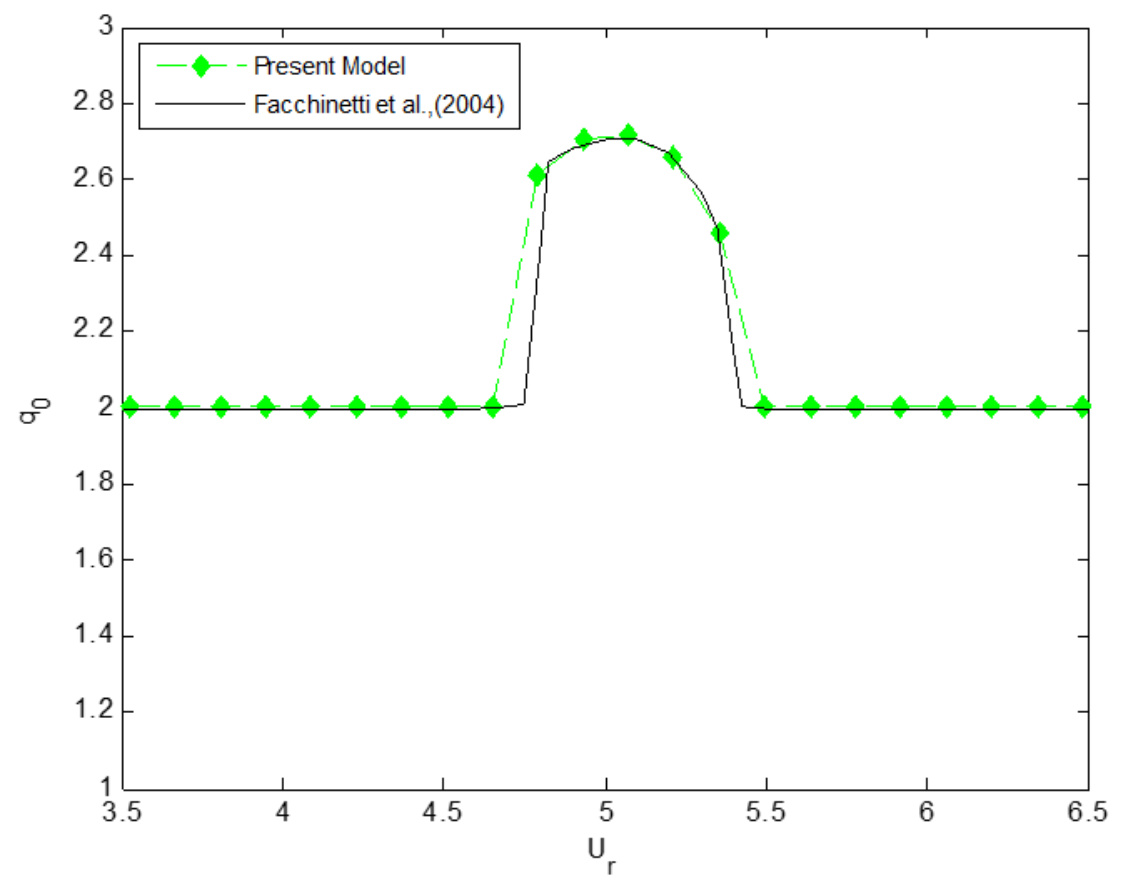

Figure 4. Amplitude of wake oscillator, $q_{0}$ using acceleration model as a function of reduced velocity, $U_{r}$ [23].

Lock-in condition is to provide the magnification of the structure motions and the structure lock-out is at rest at, $y_{0} \leq 1$, as shown in Figure 3 while, the wake oscillator sets itself on the limit cycle of amplitude $q_{0}=2$ and lift magnification is successful during lock-in in Figure 4. This system considers that a decrease in the wake oscillation amplitude can increase the structure of oscillation amplitude [23] [34].According to Sumer \& Fredsoe [35], VIV usually occurs at the reduced velocity of $4<U_{r}<8$ 
corresponding to $S_{t}=0.2$, a typical response for the VIV phenomenon. Lock-in phenomenon is observed in VIV where the shedding process is being greatly affected by the motion of the elastic structure. When the frequency of the energy input to the system due to fluctuating vortex approaches enough to the natural vibration frequency of the structure, synchronisation happens. This synchronisation effect was first reported by Bishop \& Hassan [30]. In the synchronisation range, the frequency of the vortex shedding locks at the structural motion frequency (forcing frequency) and the magnitude of the fluid force on the structure abruptly increases. This can lead to structure destruction.

The results obtained from Figure 5 through solving the present coupling model by using MATLAB and by comparing between analytical model by Facchinetti et al. [23] and experimental data obtained from Govardhan \& Williamson [12]. The results illustrated that present the coupled model is quantitatively and qualitatively in agreement with previous coupled model with the same maximum peak amplitude response $y_{0}=0.22$ and reduced velocity slightly different with present model $U_{r}=4.82$ and previous model $U_{r}=5.64$, respectively. However, the range of reduced velocity was still consistent with findings of past studies by Sumer \& Fredsoe [35], which indicated that lock-in range occurred between $4<U_{r}<8$. It can also capture the pattern and behavior of the results. However, a comparison with experimental results revealed that the present coupling model was only qualitatively in agreement but not quantitatively.

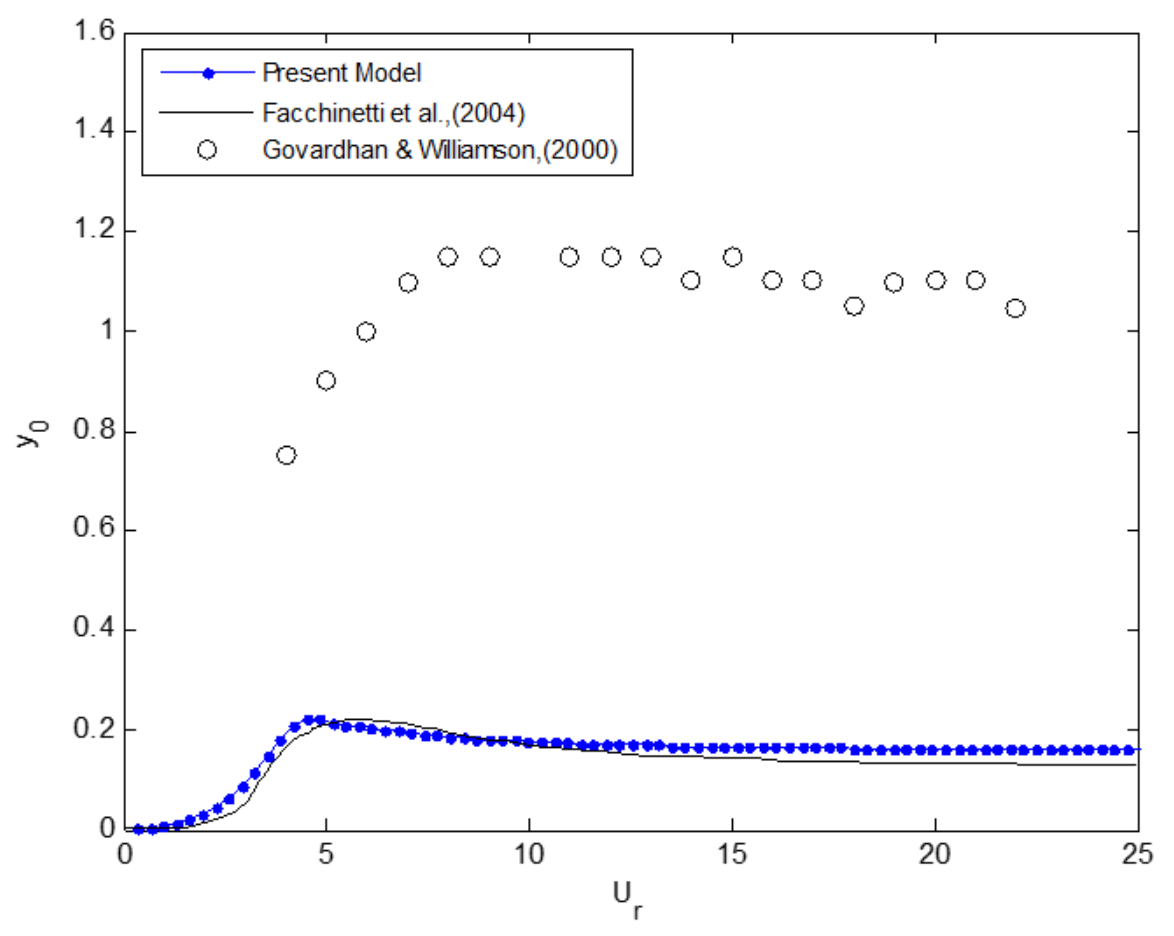

Figure 5. Oscillation amplitude response at low mass ratio $m^{*}=0.52$ and low reduced damping $\xi=0.0052$. Experimental data: o o o, [12]. Acceleration Coupled models: - , [23]. Acceleration Present coupled models: ....

In Figure 5, there are huge gaps between the present model and modified model with the experimental data. This occurred because of the 2-Dimensional (2D) and 3Dimensional (3D) aspects involved. The present coupled model in Figure 1(b) does not have length to diameter elements as compared to experimental data. Experimental data 
has length to diameter elements as shown in Figure 1(a). Present coupled model will be modified in order to capture experimental results that involved length to diameter (aspect ratio). Therefore, there is a gap between the analytical model and the experimental model. This modification will be explained in the next topic and paper related to Vortex-induced Motions (VIM) with 3D aspects involved. However, analytical model showed that the pattern and behaviour of the experimental model can be captured qualitatively. It is fair to indicate that acceleration coupling is able to represent the phenomenon of persistent lock-in and is consistent with literature [23].

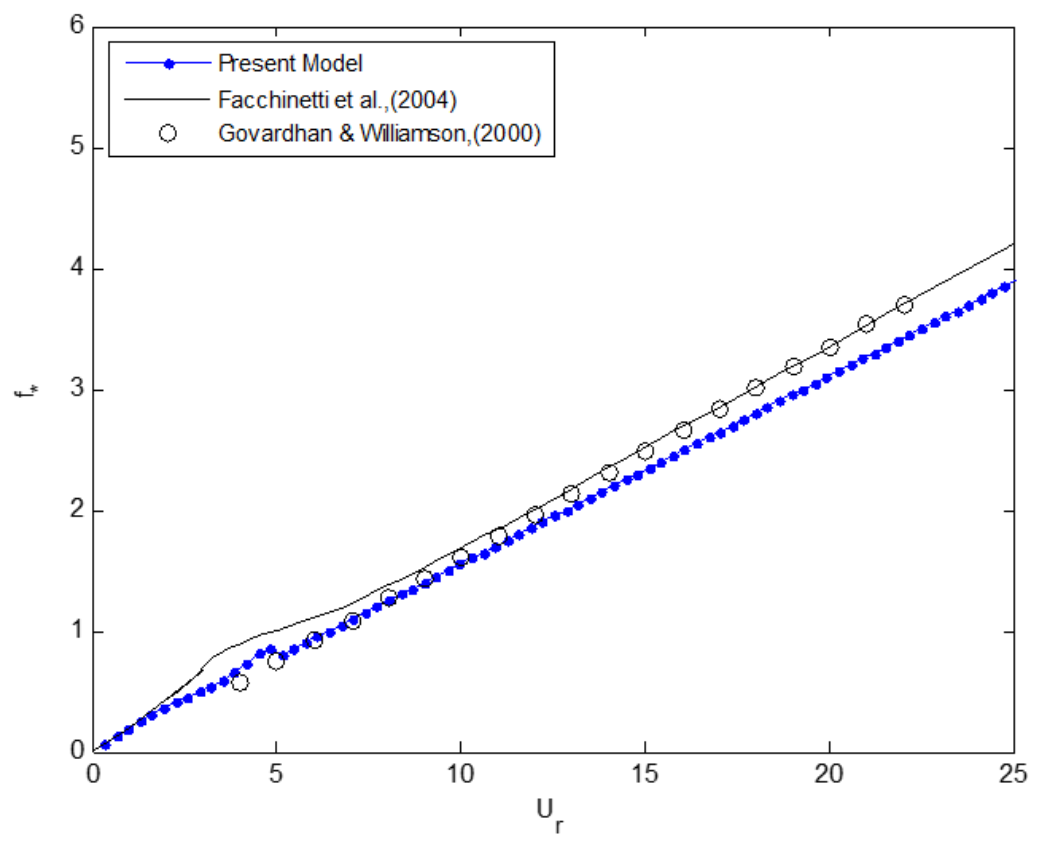

Figure 6. Angular frequency response at low mass ratio $m^{*}=0.52$ and low reduced damping $\xi=0.0052$. Experimental data: o o o, [12]. Acceleration Coupled models: - , [23]. Acceleration Present coupled models: ....

In order to be more certain on the development of angular frequency in Figure 6, where the result shows that the present coupling and coupled model with experimental data are quite consistent with $15 \%$ percentage error between present model and experimental data at approximately reduced velocity, $U_{r}=5$. When the frequency of vortex shedding is near the natural frequency of the cylinder, large amplitude motions of the cylinder may occur. These motions are called VIV and are caused by the interaction of the cylinder structure with vortices shed in the cylinder wake. Vortex shedding behind an oscillating cylinder is similar to that behind a stationary cylinder, where vortices or groups of vortices will shed at or near the Strouhal frequency. Figure 6 shows the dimensionless frequency $f^{*}=\omega / \delta$ is considered as a comparison[23].

\section{CONCLUSIONS}

In this paper, a present coupling model by using the wake oscillator model was solved by referring to analytical model from literature. This model was tested with analytical model and experimental data from literature. A comparison of analytical and experimental results were provided to give more solid proof on the present coupling model. The following conclusion can be summarized as follows: 
a) The present coupling model almost satisfy the modelling features of lock-in domain and show significant agreements with both analytical and experimental results for the pattern and behaviour results in this paper.

b) The coupling model is able to provide knowledge to understand the VIV phenomenon that is one of major problems faced by industries.

c) The present coupling model is also able to give potential means for investigations lock-in features of VIV and provide a fundamental analysis by using a reduced cost but reliable method.

d) It has shown that wake oscillator model is able to capture 3D phenomenon through 2D phenomenon and will get the better results with involved parameter of immersed length to diameter (aspect ratio).

For future work, modification of the present model will be developed to extend a better result from 2D to 3D aspect. Some future developments in the model of this paper are extending its use to a wider range of VIV and is able to significantly capture all aspects of VIV and VIM phenomenon study in marine engineering field.

\section{ACKNOWLEDGEMENTS}

Part of this research is funded by the Fundamental Research Grant Scheme (FRGS) VOT 59416 (FRGS/1/15/59416) awarded by Ministry of Higher Education Malaysia

\section{REFERENCES}

[1] Williamson Sr A, Tsay L-S, Kateeb IA, Burton" L. "Solutions for RFID Smart Tagged Card Security Vulnerabilities". AASRI Procedia. 2013;4:282-7.

[2] Zhao M, Kaja K, Xiang Y, Yan G. Vortex-induced vibration (VIV) of a circular cylinder in combined steady and oscillatory flow. Ocean Engineering. 2013;73:83-95.

[3] Rahman M, Thiagarajan K. Experiments on vortex-induced vibration of a vertical cylindrical structure: Effect of low aspect ratio. International Journal of Automotive and Mechanical Engineering. 2015;11:2515.

[4] Franzini G, Fujarra ALC, Meneghini JR, Korkischko I, Franciss R. Experimental investigation of vortex-induced vibration on rigid, smooth and inclined cylinders. Journal of Fluids and Structures. 2009;25:742-50.

[5] Facchinetti ML, De Langre E, Biolley F. Vortex-induced travelling waves along a cable. European Journal of Mechanics-B/Fluids. 2004;23:199-208.

[6] Khalak A, Williamson $\mathrm{CH}$. Motions, forces and mode transitions in vortexinduced vibrations at low mass-damping. Journal of Fluids and Structures. 1999;13:813-51.

[7] Zhao M, Cheng L, An H. Numerical investigation of vortex-induced vibration of a circular cylinder in transverse direction in oscillatory flow. Ocean Engineering. 2012;41:39-52.

[8] Sarpkaya T. A critical review of the intrinsic nature of vortex-induced vibrations. Journal of Fluids and Structures. 2004;19:389-447.

[9] Stappenbelt B, O’Neill L. Vortex-Induced Vibration of Cylindrical Structures With Low Mass Ratio. The Seventeenth International Offshore and Polar Engineering Conference: International Society of Offshore and Polar Engineers; 2007. 
[10] Modir A, Kahrom M, Farshidianfar A. Mass ratio effect on vortex induced vibration of a flexibly mounted circular cylinder, an experimental study. International journal of marine energy. 2016;16:1-11.

[11] Srinil N, Zanganeh H. Modelling of coupled cross-flow/in-line vortex-induced vibrations using double Duffing and van der Pol oscillators. Ocean Engineering. 2012;53:83-97.

[12] Govardhan R, Williamson C. Modes of vortex formation and frequency response of a freely vibrating cylinder. Journal of Fluid Mechanics. 2000;420:85-130.

[13] Gonçalves RT, Rosetti GF, Franzini GR, Meneghini JR, Fujarra ALC. Twodegree-of-freedom vortex-induced vibration of circular cylinders with very low aspect ratio and small mass ratio. Journal of Fluids and Structures. 2013;39:23757.

[14] Branković M, Bearman P. Measurements of transverse forces on circular cylinders undergoing vortex-induced vibration. Journal of Fluids and Structures. 2006;22:829-36.

[15] Billah KY, Scanlan RH. Resonance, Tacoma Narrows bridge failure, and undergraduate physics textbooks. American Journal of Physics. 1991;59:118-24.

[16] !!! INVALID CITATION !!! [3, 11, 16-21].

[17] Gabbai R, Benaroya H. An overview of modeling and experiments of vortexinduced vibration of circular cylinders. Journal of Sound and Vibration. 2005;282:575-616.

[18] Low YM, Srinil N. VIV fatigue reliability analysis of marine risers with uncertainties in the wake oscillator model. Engineering Structures. 2016;106:96108.

[19] Rahman MAA, Thiagarajan K, Leggoe J, Fitriadhy A. Wake Oscillator Model for Vortex-Induced Vibrations Predictions on Low Aspect Ratio Structures. Science and Engineering. 2015; 15.

[20] Jin Y, Dong P. A novel Wake Oscillator Model for simulation of cross-flow vortex induced vibrations of a circular cylinder close to a plane boundary. Ocean Engineering. 2016;117:57-62.

[21] Postnikov A, Pavlovskaia E, Wiercigroch M. 2DOF CFD calibrated wake oscillator model to investigate vortex-induced vibrations. International Journal of Mechanical Sciences. 2017;127:176-90.

[22] Parracho J. A Semi-Empirical Model for Vortex-Induced Vibrations of Tall Circular Towers. 2012.

[23] Facchinetti ML, De Langre E, Biolley F. Coupling of structure and wake oscillators in vortex-induced vibrations. Journal of Fluids and Structures. 2004;19:123-40.

[24] Sinsabvarodom C, Widjaja JH. The innovative hybrid Cell-Truss Spar Buoy Platform for moderate water depth. Ocean Engineering. 2016;113:90-100.

[25] Blevins RD. Flow-induced vibration. 1990.

[26] Pantazopoulos MS. Vortex-induced vibration parameters: critical review. American Society of Mechanical Engineers, New York, NY (United States); 1994.

[27] Stansby P. The locking-on of vortex shedding due to the cross-stream vibration of circular cylinders in uniform and shear flows. Journal of Fluid Mechanics. 1976;74:641-65.

[28] King R. Vortex excited oscillations of yawed circular cylinders. Journal of Fluids Engineering. 1977;99:495-501. 
[29] Griffin O. Vortex-excited cross-flow vibrations of a single cylindrical tube. Journal of Pressure Vessel Technology. 1980;102:158-66.

[30] Bishop R, Hassan A. The lift and drag forces on a circular cylinder oscillating in a flowing fluid. Proc R Soc Lond A: The Royal Society; 1964. p. 51-75.

[31] Pullin D, Saffman P. Vortex dynamics in turbulence. Annual Review of Fluid Mechanics. 1998;30:31-51.

[32] Bearman PW. Vortex shedding from oscillating bluff bodies. Annual Review of Fluid Mechanics. 1984;16:195-222.

[33] Balasubramaniam C, Goodfellow J, Price N, Kirkpatrick N. Opacification of the Hydroview H60M intraocular lens: Total patient recall. Journal of Cataract \& Refractive Surgery. 2006;32:944-8.

[34] Krenk S, Nielsen SR. Energy balanced double oscillator model for vortex-induced vibrations. Journal of Engineering Mechanics. 1999;125:263-71.

[35] Sumer B, Fredsøe J. Hydrodynamics Around Cylindrical Structures (Advanced Series on Ocean Engineering), Revised Edition, Vol. 26. World Scientific, Singapore; 2006.

\section{NOMENCLATURE}

$\begin{array}{ll}\text { VIV } & \text { Vortex-induced Vibration } \\ \text { VIM } & \text { Vortex-induced Motion } \\ \text { 1DOF } & \text { One-degree-of-freedom } \\ \text { 2D } & \text { Two-Dimensional } \\ \text { 3D } & \text { Three-Dimensional }\end{array}$

